\title{
Salon
}

\section{New thinking about postoperative delirium}

$\mathrm{T}$ he scenario is simple. An 87 year-old woman is electively admitted to hospital because of postmenopausal bleeding. The diagnosis is endometrial cancer, and the treatment is a transabdominal hysterectomy. I get involved on the first postoperative day because of the patient's delirium , which is characterized by disorientation, inattention, lethargy and no focal motor findings. Her hemoglobin, white cell count, electrolytes and troponin are all normal. I don't order a brain CT scan, but if I did, it would not be remarkable.

What's the diagnosis? In general, MEDLINE studies suggest multifactorial possibilities with medication toxicity as the prime suspect. Narcotic analgesics are usually blamed first; however, in my I5 years of experience, naloxone does not usually improve postoperative delirium. Other studies stress anticholinergics, yet cholinomimetics don't generally correct the symptoms either. Delayed clearance of anesthetics is possible, yet doesn't explain why delirium can persist for days and, in some cases, is permanent.

Textbooks are similarly unhelpful, even though they are not entirely silent on the problem of postoperative delirium. Surgical textbooks document vivid cases, but rarely explore pathophysiologic mechanisms. Anesthesia textbooks are also limited, perhaps reflecting a lack of long-term follow-up. Psychiatric textbooks focus mostly on supportive care as exemplified by good nursing and minimizing sedation. Me? I'm an internist who is left searching for an explanation.

Many of my colleagues believe delirium is caused simply by the chaotic nature of hospital wards. Perhaps the lack of clocks, excess night-time noise, unfamiliar staff and unsettling routines are enough to keep a fragile elder from thinking straight. However, this theory does not explain the patient's sudden onset of symptoms or subsequent recovery. Nor does it explain why patients admitted to the same hospital for non-

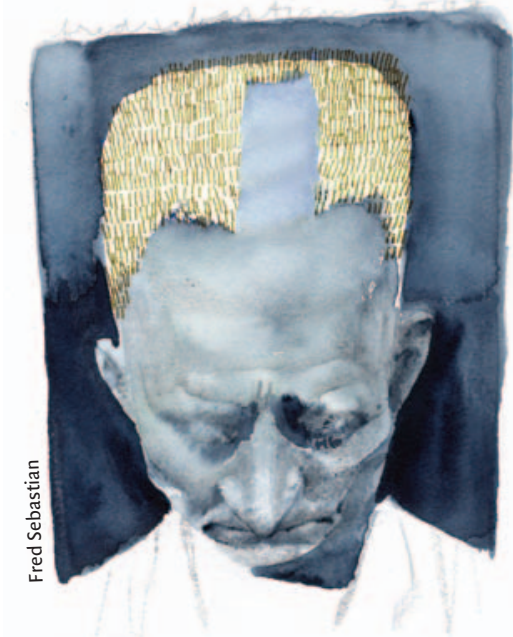

erly patients. In essence, one neuron is getting sufficient capillary flow, an immediately adjacent neuron is suffering, and this mismatch is occurring throughout the entire brain substance. This explains why each case differs, why age is the major risk factor for such delirium, why patients slowly get better and why conventional tests are normal.

An appreciation of the importance of cerebral autoregulation helps inform my clinical management of postoperative delirium. First, I don't rush since ischemic damage requires time for recovery. Second, I don't entertain unlikely diagnoses such as thiamine deficiency, myxedema coma or occult seizures. Third, I still check for other drug side effects, though more with an attitude that the damage occurred in the operating room, and that the immediate goal is to stop a bad situation from getting worse.

Avoiding postoperative delirium would require anesthetics that preserve cerebral autoregulation. However, that seems a quixotic goal given the intimate connection between neuronal activity and the surrounding capillary network. Perhaps the best approach currently is to avoid other pre-operative medications that disrupt cerebral autoregulation, although such nuances are at the frontier of current vascular medicine research. Alas, I can't think of a medication that enhances cerebral autoregulation.

\section{Donald Redelmeier MD MS(HSR) General internist}

Donald Redelmeier is a general internist at Sunnybrook Health Sciences Centre, holds the Canada Research Chair in Medical Decision Science, and is a professor at the University of Toronto, Toronto, Ont. cytes with fat stores). In contrast to resilient grasses, the tight balance between energy supply and demand leaves little margin for error in a neuron.

A failure of cerebral autoregulation triggered by anesthetics, therefore, might cause widespread neuron ischemia and clinical delirium following surgery in eld-
We encourage you to post your views on this article at cmaj.ca. Contributors are urged to read our online guidelines and send their submission (700 words maximum, French or English language) for consideration to salon@cma.ca. 